Fleischner, 2) BTS guidelines using Brock (full), 3) BTS guidelines using Brock (parsimonious).

When using the Brock model we assumed worse case scenario if clinical information was not available (i.e. family history of lung cancer present). We assumed all nodules stayed stable in size and that volumetric analysis was performed.

Results 23\% fewer patients (75 vs. 99) required surveillance when using the BTS guidelines with Brock models rather than Fleischner.

$38 \%$ fewer CT scans (132 vs. 214) would be performed in total when using Brock (full) compared with Fleischner.

$31 / 99$ patients had nodules $\geq 8 \mathrm{~mm}$. Using the Brock (full) model 9/31 would have PET-CT compared with $6 / 31$ with the Brock (parsimonious) model.

\begin{tabular}{|c|c|c|}
\hline & $\begin{array}{l}\text { No. }(\%) \text { of patients } \\
\text { requiring CT follow-up }\end{array}$ & $\begin{array}{l}\text { Total no. CT scans } \\
\text { performed in all patients } \\
\text { requiring follow-up }\end{array}$ \\
\hline Fleischner & $97(97 \%)$ & 214 \\
\hline $\begin{array}{l}\text { BTS with Brock- Full with } \\
\text { Spiculation }\end{array}$ & $75(75 \%)$ & 132 \\
\hline $\begin{array}{l}\text { BTS with Brock- } \\
\text { Parsimonious with } \\
\text { Spiculation }\end{array}$ & $75(75 \%)$ & 129 \\
\hline
\end{tabular}

Conclusion The new BTS guidelines will significantly reduce both the number of patients requiring radiological follow up for indeterminate nodules and also the total number of scans required overall.

Even assuming worst case scenario in terms of clinical risk factors there is very little difference between Brock (full) and Brock (parsimonious). This needs assessing in a larger population with clinical outcomes evaluated.

\section{P169 FDG PET-CT SCANS - WHO BEST USES THIS EXPENSIVE RESOURCE?}

${ }^{1} \mathrm{~N}$ Nwosu, ${ }^{2} \mathrm{M}$ Ledson, ${ }^{1} \mathrm{MJ}$ Walshaw. ${ }^{1}$ Royal Liverpool University Hospital, Liverpool, Merseyside; ' Liverpool Heart and Chest Hospitak, Liverpool, Merseyside

\subsection{6/thoraxjnl-2015-207770.306}

Introduction and objectives FDG PET-CT scans are a valuable tool in the diagnosis and staging of lung cancer, but their growing use in other diseases can cause resource issues, and in some cases they may be ordered by non-specialists further congesting the patient pathway. To study this further, we looked at referral patterns for PET-CT scans in the diagnosis of lung cancer at our hospital, paying particular attention to the reason for the scan and the referring clinician.

Methods A retrospective audit was undertaken of all PET-CT scans performed for the investigation of suspected lung cancer in 2014 by the Liverpool Lung Cancer Unit (LLCN). The LLCN consists of Liverpool Heart and Chest Hospital, a specialist cardiothoracic centre in the North West of England and the Royal Liverpool University Hospital, a teaching hospital.

Results All 253 PET-CT scans (186 for outpatients) carried out during 2014 for the investigation of suspected lung cancer were reviewed. One hundred and seventy five (69\%) found malignancy. Scans ordered by lung cancer specialists were more likely to demonstrate a cancer diagnosis (145 cancer versus 52 benign) compared to those requested by non-specialists (30 cancer versus 26 benign), (Chi2 $=8.21, \mathrm{P}<0.01$ ) (Figure 1 ).

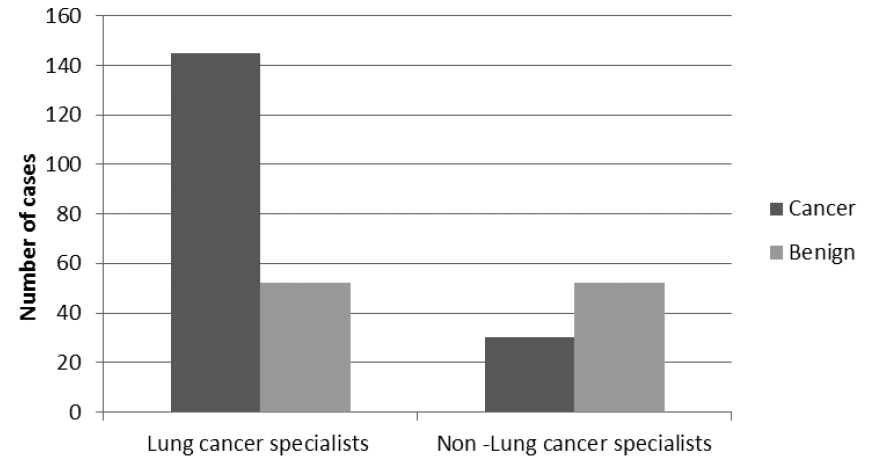

Abstract P169 Figure 1 Difference in PET-CT cancer diagnosis between lung cancer specialists and non-lung cancer specialists

The commonest causes for a negative scan result were mass 27 (34\%), nodule follow up $17(22 \%)$ and new nodule assessment $14(18 \%)$ respectively. Of 31 patients (39\%) who had a PET-CT scan for nodule assessment, $8(26 \%)$ were $<10 \mathrm{~mm}$ in diameter. Overall, $22 \%$ of all negative scans were for nodule surveillance.

Conclusions We have shown that scans arranged by lung cancer specialists are more likely to give a positive yield, and also that the guidance for their use in nodule investigation is not being strictly followed. The results indicate that there is a need for better guidelines and patient pathways to ensure the most appropriate use of expensive resources.

\section{P170 OUTCOMES FOLLOWING PURSUIT OF A TISSUE DIAGNOSIS IN ELDERLY PATIENTS WITH SUSPECTED LUNG CANCER}

${ }^{1} \mathrm{~S}$ Crawley, ${ }^{2} \mathrm{~B}$ Butler, ${ }^{3} \mathrm{G}$ Lumsden, ${ }^{3} \mathrm{~N}$ O'Rourke, ${ }^{1} \mathrm{~S}$ Sheridan, ${ }^{1} \mathrm{M}$ Sproule, ${ }^{1} \mathrm{C}$ Carlin. ${ }^{1} \mathrm{NHS}$ Greater Glasgow \& Clyde, Glasgow, UK; ${ }^{2}$ Golden Jubilee National Hospital, Clydebank, UK; ${ }^{3}$ Beatson Oncology Centre, Glasgow, UK

\subsection{6/thoraxjnl-2015-207770.307}

Background $40 \%$ of cases of lung cancer are diagnosed in patients aged 75 years and over. Comorbidities, frailty and poorer tolerance of therapy are challenges for diagnosis and management. We explored clinical outcomes and survival in a cohort of elderly patients in which a tissue diagnosis was pursued.

Methods 43 patients aged 75 and older who attended between 01/11/2013 and 30/04/2014, where a West Glasgow MDT decision was made to pursue tissue diagnosis were identified from audit data. Survival was reviewed at April 2015.

Results Mean age was $80 \pm 4$ yrs; 21 male/22 female; WHO Performance Status (PS) of 0 (2 pts), 1 (27 pts), 2 (5 pts), 3 (5 pts), Not Documented (4 pts). 17 patients had Bronchoscopy +/- EBUS-TBNA, 19 CT-guided lung biopsy, 5 other core biopsy, 2 positive pleural aspiration. Histology was non-small cell lung cancer in thirty-two (74\%) patients, small cell lung cancer in three $(7 \%)$ patients, mesothelioma in three $(7 \%)$ patients, other malignancy in $3(7 \%)$ patients and non diagnostic in 2 patients (diagnosis registered as clinical lung cancer). Seventeen (40\%) patients were treated with curative intent (surgery, radical 
radiotherapy, chemotherapy for SCLC), sixteen (37\%) patients with palliative intent (radiotherapy, chemotherapy, brachytherapy), and ten (23\%) patients received best supportive care (BSC) only. Of the five patients with PS 3, one received palliative radiotherapy and the other four BSC. Patients treated with curative intent had $71 \%$ survival at six months, $65 \%$ survival at 12 months: survival rates significantly higher compared with those receiving palliative treatment or BSC $(\mathrm{p}=0.02)$. There was no survival difference between palliative treatment and BSC ( $\mathrm{p}=$ 0.81) (Figure 1).

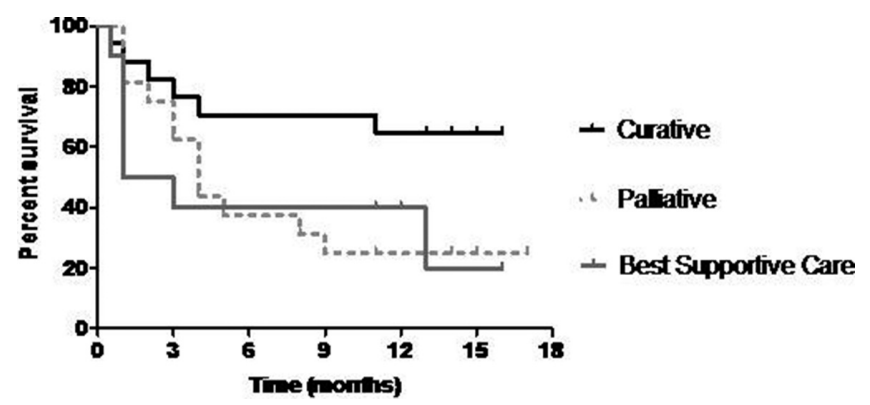

Abstract P170 Figure 1 Kaplan-Meier curves with survival stratified by treatment

Conclusion When a tissue diagnosis of lung cancer is pursued in those aged 75 and older, most patients will receive specific cancer treatment and this data informs clinical discussions about curative intent outcomes. In those who are PS 3 at baseline, BSC only is the likely outcome, and pursuing tissue diagnosis may not be appropriate.

\section{P171 A LOCAL CANCER NETWORK ROOT CAUSE AUDIT OF 62-DAY LUNG CANCER PATHWAY BREACHES}

${ }^{1} \mathrm{O}$ Eneje, ${ }^{1} \mathrm{~N}$ Kumar, ${ }^{2} \mathrm{D}$ Powrie, ${ }^{1} \mathrm{~B}$ Yung, ${ }^{3} \mathrm{M}$ Lawson. ${ }^{1}$ Basildon University Hospital NHS Foundation Trust, Basildon, UK; ${ }^{2}$ Southend University Hospital NHS Foundation Trust, Southend, UK; ${ }^{3}$ Broomfield Hospital, Chelmsford, UK

\subsection{6/thoraxjnl-2015-207770.308}

Introduction Over several years there has been an ongoing rise in 62-day Lung Cancer pathway referrals initiated by GPs as two-week wait referrals ( $2 \mathrm{WW})$. This was particularly marked in 2014 and breach rates increased across the East of England SCN. Within the Essex Lung Cancer Network an audit of these breaches was undertaken by three Trusts to look for common themes and to share best practice.

Methods Data were collected for all pathways that failed to meet the 62-day target across three NHS Trusts in Essex to identify any predictive factors for breaching the pathway. A standard proforma was used for abstraction. Results were analysed using GraphPad Prism 6 (La Jolla, CA).

Results In 2014 a total of 1,419 2WW referrals were received by the three Trusts of which $13-23 \%$ were diagnosed with lung cancer. Between 19\% and 54\% breached the 62-day target (89 of 246 pathways). The median length of the breached pathways varied from $86-88$ days by Trust. Trusts did not appear to differ significantly by end treatment after pathway breach. There were generic common themes within the breached pathways of each Trust but for the two worst performing Trusts specific pathway issues were identified. In one Trust it was clear that time delays to perform CT guided lung biopsies with a 2.75 relative risk of breaching if a pathway involved a CT biopsy (95\% CI 1.6-4.6, $\mathrm{p}<0.0001)$. At another Trust a high proportion of breached pathways had a bronchoscopy as the first test but went on to have further diagnostic biopsies by other methods.

Conclusion Many of the diagnostic delays were due to complex patient pathways needing multiple diagnostic tests. However for two Trusts significant problems were highlighted for targeted quality improvement plans. Selecting the best test to give diagnostic and staging information is vital particularly when services are stretched and capacity is reached.

\section{P172 TRAINING NURSES IN SAMPLING AND ACQUISITION OF SPECIMEN DURING EBUS GUIDED TRANSBRONCHIAL NEEDLE ASPIRATION}

V Johnson, W Stables, S Binu, C Smyth, M Walshaw, K Mohan. Liverpool Heart and Chest Hospital, Liverpool, UK

10.1136/thoraxjnl-2015-207770.309

Introduction EBUS has now become the standard of choice for mediastinal staging and in the diagnosis of paratracheal and peribronchial lesions. EBUS is usually performed as a 2 person procedure, one to locate and align the bronchoscope and a second to carry out node sampling. Whilst these have traditionally both been medical personnel, with the increase in use of this procedure and alterations in the training and availability of medical staff, we wished to look at the feasibility of empowering nurses to perform needle aspiration during EBUS.

Methods We provide a regional service for EBUS, carrying out over 420 procedures per year, often in technically difficult cases where local hospital EBUS has been unhelpful. From January 2015 we trained a band 5 registered nurse in needle aspiration, who randomly assisted in the procedure in 93 of 172 cases up to June 2015. We report our experience in these 93 cases.

Results These 93 patients were referred from 12 hospitals and 20 had undergone previous undiagnostic procedures (6 EBUS, 14 bronchoscopy). The mean age was 67 years (range $27-87$ ) and 50 were male. 82 were performed under local anaesthesia using lignocaine spray and intravenous midazolam (1 - $10 \mathrm{mg}$; mean $4 \mathrm{mgs}$ ) and the remaining 11 under general anaesthesia. 200 lymph nodes (2R, 4R, 4L, 7, 10R, 10L, 11R) and 13 lung lesions were biopsied. Results were as follows: Adequate samples were obtained in 99\% (91/93) and the NSCLC - NOS rate was 2\%. 31 adenocarcinoma, 10 squamous cell carcinoma, 10 small cell carcinoma, 1 NSCLC - NOS, 1 large cell neuroendocrine carcinoma, 1 soft tissue lesion (repeat EBUS showed myxoid spindle cell mesenchymal lesion), 1 breast carcinoma, 19 nonspecific benign nodes, 15 sarcoid and 2 TB. There were no complications.

Conclusion Our findings suggest that with motivated staff and adequate training, it is possible for nurses to perform needle aspiration during EBUS procedure with excellent results. This could be adopted as the method of choice by other centres as EBUS services continue to expand.

\section{P173 THE INVESTIGATION OF PATIENTS REFERRED WITH HAEMOPTYSIS VIA THE TWO WEEK WAIT SYSTEM IN SOUTHMEAD HOSPITAL, BRISTOL, UK}

J Harper, J Curran, M Plummeridge. Southmead Hospital, Bristol, UK

10.1136/thoraxjnl-2015-207770.310 